\title{
An inverse stage-shift model to estimate the excess mortality and health economic impact of delayed access to cancer services due to the COVID-19 pandemic
}

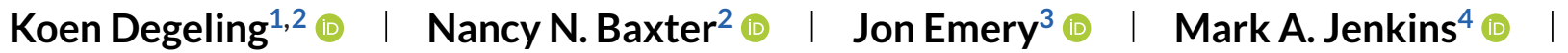

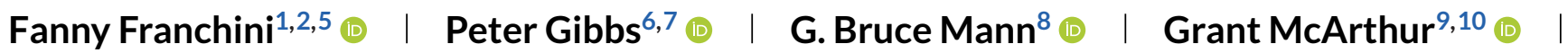 \\ Benjamin J. Solomon ${ }^{9,10}$ | Maarten J. IJzerman ${ }^{1,2,5,11}$ ( \\ ${ }^{1}$ Centre for Cancer Research and Centre for Health Policy, Faculty of Medicine, Dentistry and Health Sciences, University of Melbourne, Melbourne, Australia \\ 2 Melbourne School of Population and Global Health, Faculty of Medicine, Dentistry and Health Sciences, University of Melbourne, Melbourne, Australia \\ ${ }^{3}$ Centre for Cancer Research and Department of General Practice, Faculty of Medicine, Dentistry and Health Sciences, University of Melbourne, Melbourne, Australia \\ ${ }^{4}$ Centre for Epidemiology and Biostatistics, School of Population and Global Health, University of Melbourne, Melbourne, Australia \\ ${ }^{5}$ Department of Cancer Research, Peter MacCallum Cancer Centre, Melbourne, Australia \\ ${ }^{6}$ Division of Personalised Oncology, Walter and Eliza Hall Research Institute, Melbourne, Australia \\ ${ }^{7}$ Department Medical Oncology, Western Health, Melbourne, Australia \\ ${ }^{8}$ Department of Surgery, University of Melbourne, Melbourne, Australia \\ ${ }^{9}$ Sir Peter MacCallum Department of Oncology, University of Melbourne, Melbourne, Australia \\ 10 Department of Medical Oncology, Peter MacCallum Cancer Centre, Melbourne, Australia \\ ${ }^{11}$ Health Technology and Services Research, Technical Medical Centre, Faculty of Behavioural, Management and Social Sciences, University of Twente, Enschede, the \\ Netherlands
}

Correspondence

Dr Koen Degeling, University of Melbourne Centre for Cancer Research, Level 10,305 Grattan Street, Melbourne, 3000 VIC, Australia.

Email: koen.degeling@unimelb.edu.au

Funding information

No funding was received for performing this study.

\begin{abstract}
Aim: Decreased cancer incidence and reported changes to clinical management indicate that the COVID-19 pandemic has delayed cancer diagnosis and treatment. This study aimed to develop and apply a flexible model to estimate the impact of delayed diagnosis and treatment on survival outcomes and healthcare costs based on a shift in the disease stage at treatment initiation.

Methods: A model was developed and made publicly available to estimate populationlevel health economic outcomes by extrapolating and weighing stage-specific outcomes by the distribution of stages at treatment initiation. It was applied to estimate the impact of 3- and 6-month delays based on Australian data for stage I breast cancer, colorectal cancer, and lung cancer patients, and for T1 melanoma. Two approaches were explored to estimate stage shifts following a delay: (a) based on the relation between time to treatment initiation and overall survival (breast, colorectal, and lung cancer), and (b) based on the tumor growth rate (melanoma).

Results: Using a conservative once-off 3-month delay and considering only shifts from stage I/T1 to stage II/T2, 88 excess deaths and $\$ 12$ million excess healthcare costs were predicted in Australia over 5 years for all patients diagnosed in 2020. For a 6-month
\end{abstract}


delay, excess mortality and healthcare costs were 349 deaths and $\$ 46$ million over 5 years.

Conclusions: The health and economic impacts of delays in treatment initiation cause an imminent policy concern. More accurate individual patient data on shifts in stage of disease during and after the COVID-19 pandemic are critical for further analyses.

\section{KEYWORDS}

COVID-19, diagnostic delays, economic outcomes, health outcomes, modeling, oncology, stage shift, time to treatment initiation, treatment delays

\section{1 | INTRODUCTION}

Several countries have reported declining cancer diagnosis registrations following the COVID-19 outbreak and flagged this as an imminent policy concern, because it implies substantially delayed cancer diagnoses. For example, the Netherlands Cancer Registry has presented weekly cancer incidence estimates since the outbreak of COVID-19. At the peak of the outbreak, cancer incidence for all cancers combined was $40 \%$ lower on average compared to historical data, with the incidence rate for skin cancer decreasing $60 \%{ }^{1}$ In addition, there was an up to $66 \%$ reduction in chemotherapy administered across eight hospitals in the United Kingdom (UK). ${ }^{2}$ Delayed cancer diagnoses and treatment will result in stage shifts, where a proportion of cancers will have moved to the next phase of disease where it is more likely to be fatal. Several professional societies have flagged these observations as requiring urgent action and encouraged interventions to restore the normal diagnosis and treatment throughput as much as possible. ${ }^{3}$

The second wave, or indirect pandemic consequences, on the health service, which is not to be confused with a second wave of outbreaks of COVID-19, is referred to as the impact of delayed access to care on health outcomes. These delays, as illustrated in Figure 1 for cancer, may occur at different points throughout the care pathway. ${ }^{4}$ In this terminology, there is an expected impact for patients due to diagnostic delays, caused by delayed investigation of symptoms due to delays in general practitioner (GP) visits and delayed access to diagnostic pathways. Diagnoses can also be delayed by a suspension of, or reduced participation in, screening programs. Additionally, there may be an impact of COVID-19 on care interruptions for patients already diagnosed and being treated for cancer. For instance, care may be delayed or changed for immunocompromised patients at high risk of infection in the hospital setting. Such changes include delaying surgery, a shift from intravenously to orally administered systemic therapies, and modified radiotherapy delivery to reduce time in hospital. ${ }^{5}$

Several studies have explored the relationship between health outcomes and the time to treatment initiation (TTI), which includes both delays in diagnosis as well as treatment delays after diagnosis (Figure 1). A systematic review concluded there is a relation between diagnostic delays and poorer outcomes for some cancers, including breast, head and neck, testicular, and melanoma. ${ }^{6}$ Other studies also concluded a link between TTI and overall survival, with lung cancer and pancreatic cancer being more heavily impacted by longer TTIs compared with breast cancer, for example. ${ }^{7}$ Other recent studies concluded evidence of a relation between diagnostic delay and outcomes in head and neck cancers. ${ }^{8,9}$ For other cancers, like colorectal cancer, there is some evidence that delays to surgery of up to 30 days may not directly impact health outcomes. ${ }^{7,10}$ Despite varying levels of evidence of a relation between TTI and outcomes, studies conclude health

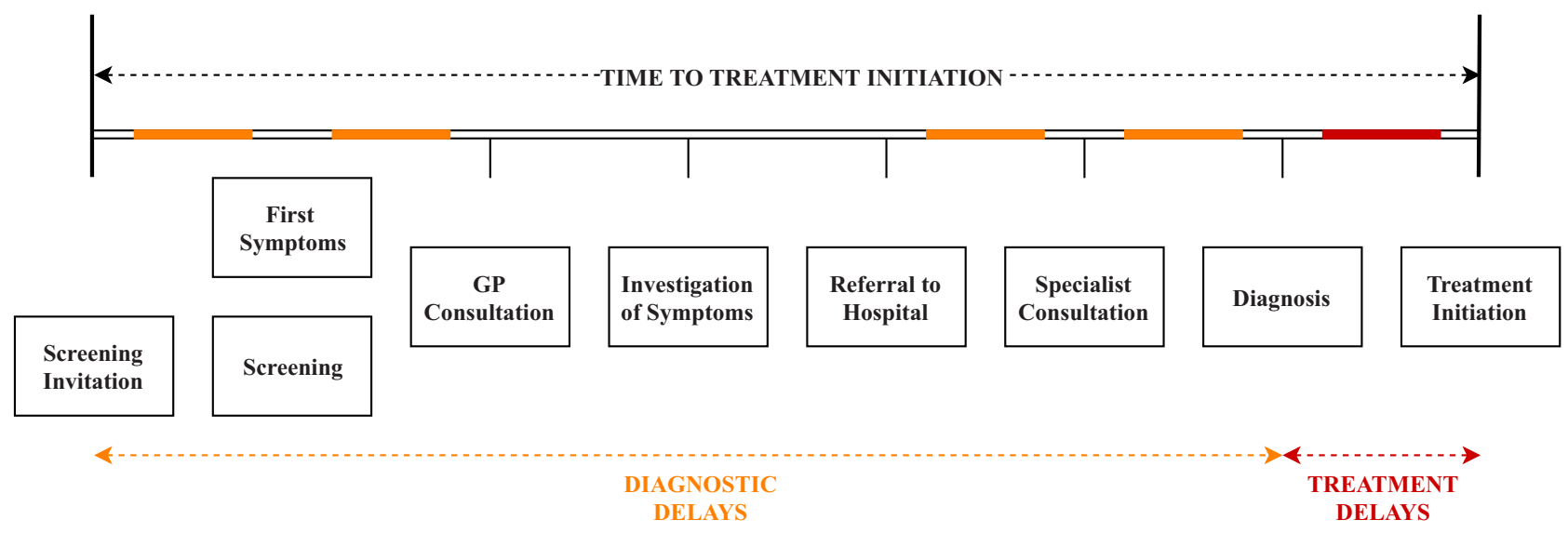

FIGURE 1 Graphical illustration of different types of delays within the care pathway that may be induced by service disruptions due to the COVID-19 pandemic [Colour figure can be viewed at wileyonlinelibrary.com] 


\section{Distribution of Stage at Treatment Initiation}

\section{Stage-specific and COMBINED Overall Survival}
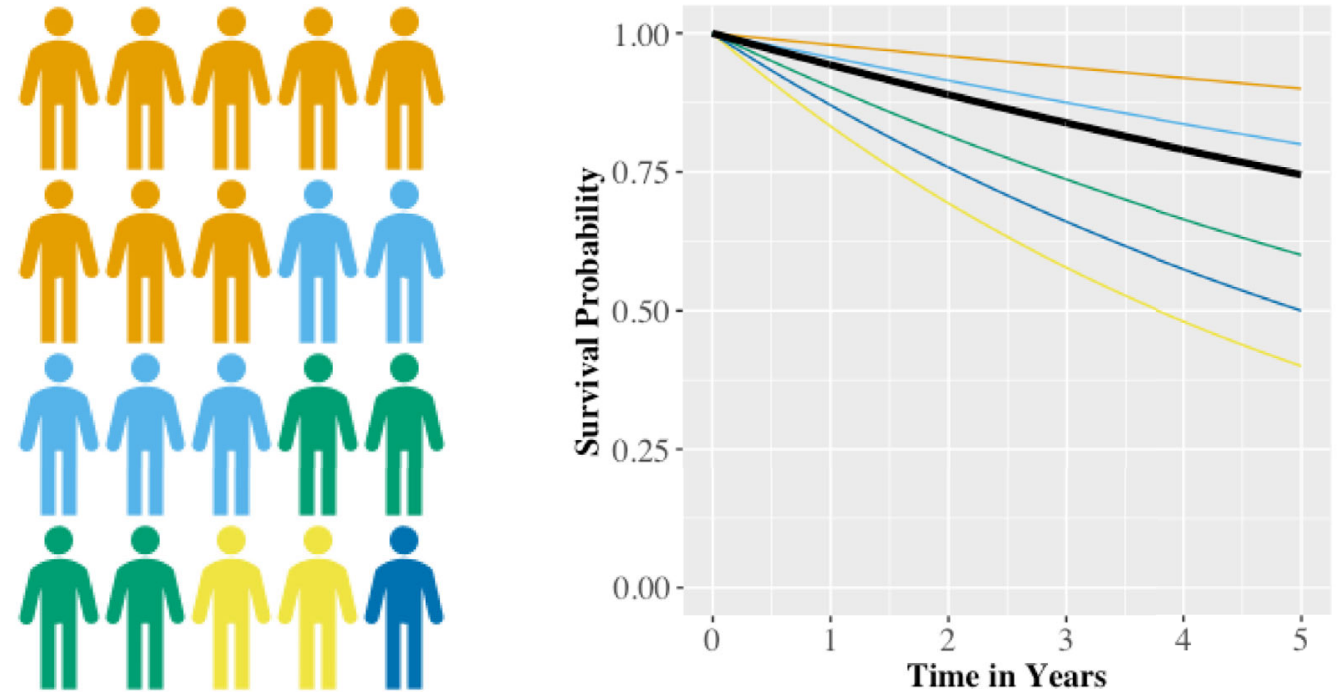

Disease Stage:

- COMBINED

- stage I

- stage II

- stage III

- stage IV

- unknown

FIG URE 2 Graphical illustration of how the combined overall survival for a patient population is obtained by weighting stage-specific survival estimates by the stage distribution at treatment initiation [Colour figure can be viewed at wileyonlinelibrary.com]

systems should focus on the effectiveness of pathways to shorten TTI. ${ }^{11}$ Considering the reported decreases in cancer diagnoses and changes to clinical management during the COVID-19 pandemic, it is anticipated that the resulting delays in TTI may result in cancer progressing to a more advanced stage by the time of treatment initiation.

We aimed to estimate the cancer-specific excess mortality due to diagnostic and treatment delays caused by COVID-19 restrictions by quantifying the impact of delays in TTI on cancer stage progression. Rather than assuming that TTI delays have a direct impact on health outcomes, this approach assumed that delays only impact outcomes if they result in disease-stage progression, i.e. treatment being initialized at a more advanced disease stage. ${ }^{12}$ The analysis was performed based on a flexible stage-shift model that estimates long-term outcomes based on the distribution of disease stage at treatment initiation. The model was illustrated by estimating the impact of COVID-19 induced TTI delays on shifts from stage I to stage II for breast cancer, colorectal cancer, and lung cancer, and from tumor stage 1 (T1) to T2 for melanoma.

\section{2 | METHODS}

The model developed to estimate the impact of delays in TTI due to the COVID-19 pandemic was based on extrapolation of the impact of changes in the distribution of disease stage at treatment initiation. It was made publicly available online using the shiny package ${ }^{13}$ in $\mathrm{R}$ (version 3.6.1) ${ }^{14}$ together with the scripts used to perform the analyses included in this manuscript: http://stage-shift.personex.nl/. Although data for any cancer type or country can be entered in the online model, Australian data for four of the country's most common cancers are considered here (breast cancer, colorectal cancer, lung cancer, and melanoma). The subsequent sections describe how the model estimates expected deaths, life years lost and healthcare costs based on the distribution of disease stage at treatment initiation, and introduce two approaches for calculating the stage shift resulting from delays in TTI. We refer to the baseline scenario as our best estimate of the distribution of disease stage at treatment initiation in a scenario without delays, and the stage shift scenario as the distribution following delays.

\subsection{Health impact in terms of expected life years}

The expected survival in life years for a particular distribution of cancer stages at treatment initiation was calculated based on the area under the combined survival curve. This combined survival curve was obtained by weighting stage-specific survival probabilities by the distribution of disease stage at treatment initiation (Figure 2). To allow for appropriate interpolation between data points and extrapolation beyond the time horizon of the data, parametric distributions were fitted to the combined survival data using nonlinear least-squares regression of their parameters on logarithmic scale: exponential, Gamma, Gompertz, log-Normal, log-logistic, and Weibull. In absence of individual patient data, the best-fitting distribution was selected based on visual inspection. The impact of a stage shift on the expected survival was calculated as the difference between the survival outcomes for the baseline scenario compared to those of the stage shift scenario.

To illustrate the framework, disease stage-specific (TNM stage) relative survival probabilities were taken from the 2019 "Cancer data in Australia" report by the Australian Institute of Health and Welfare 
$(\mathrm{AlHW})^{15}$ for breast cancer, colorectal cancer, and lung cancer (Table 1). For melanoma, tumor ( $\mathrm{T})$ stage-specific overall survival probabilities were estimated from the Melanoma Research Victoria (MRV) registry using data on 2,521 patients with a median follow-up of 3.4 years (Table 1). For this analysis, expected survival was estimated for time horizons of 5 and 10 years by extrapolating combined year-specific survival probabilities using Gompertz (breast cancer and melanoma) and Gamma (colorectal and lung cancer) distributions. Patient-level estimates of the expected life years were translated into population-level estimates based on the predicted 2020 incidence for Australia. ${ }^{15}$

\subsection{Economic impact in terms of healthcare costs}

The expected healthcare costs were calculated as the stage-specific costs weighted according to the distribution of disease stage at treatment initiation. In the illustration, the impact of stage shifts on healthcare costs in Australia was estimated up to 5 years after diagnosis from a public healthcare payer perspective. Goldsbury et al used data of 7624 participants with a cancer diagnosis registered in the New South Wales Cancer Registry between 2006 and 2010, matched to 22661 cancer-free controls, to determine excess healthcare costs of the 10 most common cancers in Australia. ${ }^{16}$ Healthcare costs considered included those associated with inpatient hospital episodes, emergency department presentations, medicines captured in the Pharmaceutical Benefits Scheme (PBS), and medical services captured in the Medicare Benefits Schedule (MBS). Goldsbury et al. did not publish disease stage-specific costs that were needed to estimate the impact of stage shifts. Therefore, stage-specific costs were approximated based on these data for each cancer separately in three steps (see Supplementary Material for details). All costs were indexed to 2020 Australian dollars (\$) using the Australian Health Index. ${ }^{17}$

\subsection{Distribution of disease stage at treatment initiation}

For this analysis, data on the distribution of disease stage published by the AlHW ${ }^{15}$ were used to define the baseline scenario for breast, colorectal, and lung cancer, and data published by Cancer Council Victoria for the distribution of T stage for melanoma. ${ }^{18}$ In the absence of empirical evidence on the distribution of disease stage at treatment initiation during the COVID-19 pandemic, 2 approaches were explored and compared to approximate this distribution through estimating time to stage progression. Two realistic delay periods were considered: 3 months and 6 months. These periods were selected because they were considered plausible from a clinical perspective and have been used in a previous modeling study. ${ }^{3}$ In absence of real-world data on delays for patients with advanced disease stages and based on the belief that those patients are more likely to seek care regardless of restrictions (e.g., through emergency presentations), the analyses were selected to be conservative in that they only consider shifts from stage I to stage II for breast, colorectal and lung cancer, and from stage T1 to T2 for melanoma. The model and online tool, however, can also be used to estimate the health economic impact of shifts to more advanced and metastatic disease, but such scenarios were not presented because of the before mentioned reasons.

The treatment delay approach was based on the relation between TTI and survival, estimating how long a delay in TTI needed to be such that the mortality rate for stage I patients matches the rate for stage II patients. This approach was applied for breast cancer, colorectal cancer, and lung cancer based on hazard ratios estimated in a large population study. ${ }^{7}$ This study used data from the National Cancer Database (NCDB) (breast cancer: $\mathrm{n}=1368$ 024; colorectal cancer: $\mathrm{n}=662$ 094; lung cancer: $n=363863$ ) to estimate hazard ratios for delays beyond 6 weeks. The time to stage progression estimates were used to calculate the proportion of patients who would experience a stage progression in each month in which services were disrupted, assuming incident cases are diagnosed evenly throughout the year. These proportions were then applied to the baseline scenario to define the stage shift scenario for the 3- and 6-month delays separately. Details are provided in the Supplementary Material.

The tumor growth approach estimated the proportion of patients who would progress to a more advanced stage based on the tumor growth rate. This approach was applied for melanoma, where the $T$ stage is directly linked to the thickness of the tumor (Breslow thickness) and evidence on the monthly increase of tumor thickness is available. ${ }^{19}$ Based on the growth rate and the distribution of tumor thickness without delays estimated from the MRV registry, it was simulated how many patients would have progressed to a T2 tumor following a 3 or 6-month delay. The estimated proportions were applied to the baseline scenario to define the stage shift scenarios. Details are provided in the Supplementary Material.

\section{3 | RESULTS}

Table 2 presents an overview of the results of the analyses performed using the stage-shift framework for a 3- and 6-month delay in TTI for the 2020 Australian incident breast cancer, colorectal cancer, lung cancer, and melanoma patient populations. Note that in Table 2, the probability of progression represents the probability that any stage I/T1patient would progress to stage II/T2 following a 3- or 6-month delay. The percentage of patients progressing represents the proportion of patients diagnosed in 2020 who will consequently experience a stage shift, which is calculated based on the probability of progression and the duration of service disruptions. Hence, the percentage of patients progressing is one-quarter of the probability of progression for a 3 -month delay (ie, a quarter of a year) and half the probability of progression for a 6-month delay (ie, half a year).

For a complete delay in TTI of 3 months for all stage I breast cancer patients across Australia, the treatment delay approach estimated a $5.6 \%$ probability that these patients would have progressed to stage II during this period of disruption. For a 6-month delay, this probability 
TAB LE 1 Overview of the cancer type and stage-specific data used for estimating the impact in terms of survival and 5-year healthcare costs, with TNM classification and relative survival probabilities for breast, colorectal, and lung cancer, and T-stage classification and overall survival probabilities for melanoma

\begin{tabular}{|c|c|c|c|c|}
\hline & Breast Cancer & Colorectal Cancer & Lung Cancer & Melanoma \\
\hline \multicolumn{5}{|l|}{ Stage at Treatment Initiation (\%) } \\
\hline stage II / T2 & 34.7 & 24.3 & 6.5 & 13.6 \\
\hline stage III / T3 & 12.1 & 23.6 & 11.2 & 10.2 \\
\hline stage unknown & 5.5 & 12.3 & 28.5 & 7.8 \\
\hline \multicolumn{5}{|l|}{ 1-year survival (\%) } \\
\hline stage I / T1 & 100.0 & 99.3 & 90.8 & 99.2 \\
\hline stage II / T2 & 99.9 & 96.4 & 69.8 & 98.0 \\
\hline \multicolumn{5}{|l|}{ 2-year survival (\%) } \\
\hline stage I/T1 & 100.0 & 99.1 & 82.7 & 97.9 \\
\hline stage II / T2 & 98.8 & 94.2 & 52.4 & 94.5 \\
\hline stage III / T3 & 93.4 & 85.5 & 32.9 & 89.5 \\
\hline stage IV / T4 & 57.6 & 32.0 & 8.3 & 65.0 \\
\hline stage unknown & 83.1 & 65.8 & 27.1 & 80.7 \\
\hline \multicolumn{5}{|l|}{ 3-year survival (\%) } \\
\hline stage I/T1 & 100.0 & 98.8 & 76.1 & 96.9 \\
\hline stage II / T2 & 95.7 & 90.8 & 35.6 & 85.8 \\
\hline stage III / T3 & 84.2 & 74.3 & 20.1 & 78.8 \\
\hline stage IV / T4 & 38.7 & 16.8 & 3.9 & 67.5 \\
\hline stage unknown & 69.9 & 58.7 & 16.1 & 74.6 \\
\hline \multicolumn{5}{|l|}{ 5-year survival (\%) } \\
\hline stage I/T1 & 100.0 & 98.6 & 67.7 & 91.7 \\
\hline stage II / T2 & 94.6 & 88.6 & 32.3 & 83.0 \\
\hline stage III / T3 & 80.6 & 71.3 & 17.1 & 73.2 \\
\hline stage IV / T4 & 32.0 & 13.4 & 3.2 & 61.6 \\
\hline stage unknown & 65.0 & 56.9 & 14.4 & 73.7 \\
\hline \multicolumn{5}{|l|}{ Healthcare costs over 5 years } \\
\hline stage I/ T1 & $\$ 50,699$ & $\$ 51,531$ & $\$ 28,532$ & $\$ 13,981$ \\
\hline stage II / T2 & $\$ 67,069$ & $\$ 66,504$ & $\$ 28,532$ & $\$ 25,715$ \\
\hline stage III / T3 & $\$ 98,632$ & $\$ 121,558$ & $\$ 67,065$ & $\$ 30,644$ \\
\hline stage IV / T4 & $\$ 105,934$ & $\$ 120,015$ & $\$ 79,045$ & $\$ 38,216$ \\
\hline stage unknown & $\$ 100,860$ & $\$ 121,077$ & $\$ 70,678$ & $\$ 32,960$ \\
\hline
\end{tabular}


TA B LE 2 Results of the exploratory analyses for the 2020 Australian incident populations, only considering shifts from stage I to stage II (breast cancer, colorectal cancer and lung cancer) and stage T1 to stage T2 (melanoma)

\begin{tabular}{|c|c|c|c|c|c|c|c|c|}
\hline & \multicolumn{2}{|c|}{ Breast Cancer } & \multicolumn{2}{|c|}{ Colorectal Cancer } & \multicolumn{2}{|c|}{ Lung Cancer } & \multicolumn{2}{|c|}{ Melanoma } \\
\hline $\begin{array}{l}\text { Probability of } \\
\text { progression from I } \\
\text { to II or T1 to T2 }\end{array}$ & $5.6 \%$ & $10.9 \%$ & $3.0 \%$ & $5.9 \%$ & $8.3 \%$ & $16.0 \%$ & $32.0 \%$ & $64.0 \%$ \\
\hline \multicolumn{9}{|l|}{ Health Outcomes } \\
\hline $\begin{array}{l}\text { Excess deaths after } 5 \\
\text { years }\end{array}$ & 7 & 25 & 3 & 11 & 11 & 43 & 67 & 270 \\
\hline $\begin{array}{l}\text { Life years lost over a } \\
\text { 5-year time } \\
\text { horizon }\end{array}$ & 20 & 64 & 7 & 29 & 44 & 170 & 195 & 791 \\
\hline \multicolumn{9}{|l|}{ Economic Outcomes } \\
\hline $\begin{array}{l}\text { Incremental } \\
\text { healthcare costs } \\
\text { (in thousands)* }\end{array}$ & $\$ 1,981$ & $\$ 7,699$ & $\$ 411$ & $\$ 1,619$ & - & - & $\$ 9,109$ & $\$ 36,436$ \\
\hline
\end{tabular}

*No differences between treatment costs for stage I compared to stage II were assumed for lung cancer.

was estimated to be $10.9 \%$. Because not all stage I patients in the 2020 population would have been diagnosed in the months where COVID19 restrictions were imposed, the proportions of stage I patients diagnosed in 2020 experiencing progression were $1.4 \%$ and 5.5\%, for a 3and 6-month delay, respectively. The stage-shift model then estimated this would result in 7 or 25 excess deaths after 5 years for the 2020 breast cancer population, respectively. Over a 5- and 10-year time horizon, the estimated expected loss in life years for the 2020 population was 20 and 77 life years if a 3-month delay was observed, and 64 and 239 life years for a 6-month delay. Following the stage-shift due to a 3-month delay, healthcare costs for the incident 2020 breast cancer population were estimated to increase by $\$ 2.0$ million, whereas a $\$ 7.7$ million increase was estimated for a 6-month delay.

Because the time to stage progression estimated using the treatment delay approach for colorectal cancer exceeded that of breast cancer (see Supplementary Material), the probability of stage progression was lower compared to breast cancer at 3.0\% for a 3-month delay and $5.9 \%$ for a 6-month delay. This translated to proportions of the 2020 incident stage I population of $0.7 \%$ and $2.9 \%$, respectively. The number of excess deaths after 5 years was estimated to be 3 and 11, for a 3 and 6-month delay, respectively. Over a 5 and 10-year time horizon, the loss in expected survival was 7 and 24 life years for a 3-month delay, and 29 and 96 life years for a 6-month delay. The increment in healthcare costs for a 3-month delay in the 2020 diagnosed population was estimated at $\$ 411000$, with an estimated increase of $\$ 1.6$ million for a 6-month delay.
For lung cancer, the probability of progression during a 3-month delay was estimated to be $8.3 \%$ according to the treatment delay approach, and $16.0 \%$ for a 6-month delay. Consequently, the proportion of stage I lung cancer patients from the 2020 population that would progress during the delay was $2.1 \%$ and $8.0 \%$, for a 3 and 6-month delay, respectively. Given the more substantial difference in overall survival between stage I and stage II for lung cancer compared to breast and colorectal cancer, this translated into estimated excess deaths of 11 and 43 after 5 years, respectively. In terms of expected survival in life years, a 3-month delay was estimated to result in a loss of 44 and 98 life years, and a 6-month delay to result in a loss of 170 and 373 life years, over a 5 and 10-year time horizon, respectively. No differences in healthcare costs for treating the incident 2020 lung cancer population following a stage shift from stage I to stage II were found, because treatment costs for these two stages were estimated to be equal (Table 1).

Melanoma was the only tumor stream for which stage progression following a delay in TTI was estimated using the tumor growth approach, which contributed to the highest impact across all considered cancer types. The probabilities of progressing due to a 3 or 6-month delay were $32.0 \%$ and $64.0 \%$, respectively, which was estimated to result in $8.0 \%$ or $32.0 \%$ of the $2020 \mathrm{~T} 1$ melanoma patients progressing to T2 disease. At 67 following a 3-month delay and 270 following a 6month delay, the number of excess deaths after 5 years was the highest for melanoma. This translated into 195 or 626 life years lost for a 3-month delay, and 791 or 2584 life years lost for a 6-month delay, over 
a 5 and 10-year time horizon, respectively. Based on the stage shift, healthcare costs for treating the 2020 diagnosed T1 melanoma population were estimated to increase with $\$ 9.1$ million following a 3-month delay or $\$ 36.4$ million following a 6-month delay.

\section{DISCUSSION}

Here we present an early modeling study to estimate excess mortality due to delays in TTI as a result of the COVID-19 outbreak. For the 4 cancers included, we estimated the excess mortality projected across Australia due to the expected fraction of cancers transitioning from stage I/T1 to stage II/T2 disease. Excess mortality of 88 deaths after 5 years was estimated for a delay of 3 months, and 349 deaths for a delay of 6-months. In addition, excess healthcare costs can be up to $\$ 12$ million over 5 years for a 3 -month delay, increasing to $\$ 46$ million for a 6-month delay. This estimated excess mortality causes an imminent policy concern. If new cancer diagnoses continue to remain low compared to baseline and further delays occur if the hospital and surgical capacity are affected, the long-term effect of COVID-19 on cancer health outcomes should not be neglected.

This study is not the first to flag concerns of increasing diagnostic and treatment delays. A rapid decline in cancer incidence in the Netherlands has led to immediate awareness campaigns that encouraged people to see their GP if they experienced suspicious symptoms. ${ }^{1}$ Earlier studies have also flagged the collateral damage occurring from declining cancer incidence and reductions in delivery of multi-disciplinary cancer services in the UK. ${ }^{2,3}$ Lai et al. used hospital admission data to estimate excess deaths of about 6270 in the UK and 33890 in the United States in addition to COVID-19 related mortality. ${ }^{2}$ Sud et al. used hazard ratios for the impact of delayed surgery on overall survival, estimating attributable deaths of 10555 in the UK resulting from a 6-month delay. ${ }^{3}$ Most recently, Maringe et al. utilized linked English National Health Service (NHS) cancer registration and hospital administrative datasets in a modeling study to estimate excess mortality between 281 and 344 deaths for breast cancer, 1445-1563 for colorectal cancer, 1235-1372 for lung cancer, and 330-342 for oesophageal cancer. ${ }^{20}$ Differences between findings from these studies and our conservative estimates can be explained by three factors. First, health care systems and population sizes and demographics are different between the UK, the United States, and Australia. Second, our stage-shift framework assumed delays only have an impact on health outcomes if they result in progression to a more advanced disease stage, whereas the other studies assumed an impact for every delayed patient. Third, we only considered shifts from stage I to stage II or from T1 to T2 in our illustration, whereas the other studies considered all disease stages to be subject to delays. As a consequence, our estimates on the excess number of cancer deaths will likely underestimate the actual number of excess cancer deaths. However, it is important to note that additional analyses, including those of shifts in more advanced disease stages and for different countries, can be performed using the online version of the model.
While the stage-shift model is flexible and easy to implement, one criticism of the approach is that stage at treatment initiation is assumed to be an ordinal variable for which survival and costs were extrapolated. However, there is significant heterogeneity within each stage between patients with poorer and better prognoses (eg, tumor size of $4 \mathrm{~mm}$ compared to $17 \mathrm{~mm}$ in breast cancer). Nevertheless, compared to previous modeling approaches, a strength of the current model is that it does not assume that the delays will affect all patients, but only those whose disease progresses to a subsequent, more advanced stage. This is an essential feature, as current observational studies and systematic reviews are not conclusive about the relation between diagnostic delays and survival and significant heterogeneity is expected between cancers and between individual patients. ${ }^{6}$

Because patient-level data about the disease stage at treatment initiation during the COVID-19 pandemic is not yet available, we estimated this distribution based on the delay in TTI. For this estimate, we extensively searched the literature for observational studies investigating the relationship between treatment delay, stage progression, and survival, ${ }^{7,21-23}$ as well as mathematical models predicting stage progression from tumor growth. ${ }^{24,25}$ The likelihood of stage shifts according to the treatment delay approach strongly depends on the assumed hazard ratios for the impact of delays in TTI on survival. In the illustration for stage I breast cancer, colorectal cancer, and lung cancer, these hazard ratios were extracted from a recent study that used a large patient sample from the NCDB to obtain estimates for multiple cancer types according to a consistent analysis framework. Although these estimates from a single study improve comparability between cancer types, other studies have also estimated the impact of delays in TTI on survival. For breast cancer, Bleicher et al. (2016) used data from the NCDB ( $n=115790$ ) and Surveillance, Epidemiology and End Results (SEER) database $(n=945441)$ to estimate hazard ratios of 1.16 (NCDB) and 1.13 (SEER) for every 30-day increase in time between diagnosis and surgery for stage $1 .{ }^{22}$ These estimates are higher compared to the hazard ratio of 1.018 for a 7-day delay used here, which translates to a 30-day hazard ratio of 1.08. For colorectal cancer, Lee et al. (2019) used data on 39900 patients from the Taiwan Cancer Registry Database to estimate a hazard ratio of 1.41 for TTI between 31 and 150 days compared to TTI $<31$ days for stage I, and a hazard ratio of 2.66 for TTI beyond 151 days, ${ }^{23}$ which are substantially higher than the hazard ratio used in the illustration. Regarding lung cancer, Samson et al. used data on 39995 stage I non-small cell lung cancer patients from the NCDB to estimate a hazard ratio of 1.004 for every week between diagnosis and surgery beyond 8 weeks, ${ }^{26}$ which is lower than the hazard ratio of 1.032 used here.

While different assumptions had to be made, we aimed to provide conservative but realistic estimates based on the Australian data and by analyzing a realistic 3-month and a possible 6-month delay. First, although the impact of stage progressions from stage II to stage III and from stage III to stage IV are likely to be associated with significant excess mortality and cost, we only considered shifts from stage I to stage II disease for breast, colorectal, and lung cancer, and shifts from T1 to T2 for melanoma. These shifts have a relatively limited impact on expected survival and healthcare expenditure. Second, because the 
illustration focusses on stage I and T1 cancers, only a proportion of the complete patient populations was considered to experience delays and be at risk of disease progression: $62 \%$ for melanoma, $43 \%$ for breast cancer, $22 \%$ for colorectal cancer, and $12 \%$ for lung cancer (Table 1). Since diagnostic and treatment delays are likely to occur throughout all stages of disease, the estimates in our illustration are relatively conservative, because those patients would also be at risk of disease progression. Diversity in the proportion of stage I or T1 patients between the 4 cancers relative to their absolute incidence also explains differences in excess mortality, with the impact of breast cancer and melanoma being more severe. If we had modeled additional stage shifts from stage III to stage IV, for example, the excess mortality in lung and colorectal cancer would have been substantially higher. Third, we only model incident cases, i.e. patients diagnosed in 2020. But delays in treatment for prevalent patients, such as surgery, have also been reported. Furthermore, it is likely that prevalent cancer patients at high-risk for COVID19 will experience additional service disruptions, such as adjusted therapy intensity. Obviously, when considering the impact of treatment delays and modifications for prevalent patients, real-world excess mortality is expected to be substantially higher. Fourth and finally, the economic impact of stage shifts was modeled based on an average cost per diagnosis conditional on the stage at diagnosis. Consequently, the cost of increased cancer-related deaths, for example in terms of palliative care, was not reflected. If the death-related cost would have been reflected separately, the impact of the delays on economic outcomes would have been even more profound. Therefore, the estimate of the economic impact can be considered conservative in that regard.

In conclusion, we have developed a flexible stage-shift model allowing estimation of the long-term health outcomes and economic impact of delays in access to care due to the COVID-19 pandemic. Initial analyses have estimated excess mortality of nearly 90 deaths and excess costs of more than $\$ 12$ million based on a realistic 3-month delay. However, the analyses also demonstrate that hundreds of life years may be lost over the following years due to delays in TTI if delays increase to 6 months. Although the results are preliminary, they are indicative of the potential impact of service disruptions due to COVID-19 on cancer outcomes in the years to come and can create awareness and provide an incentive for restoring health service throughput levels to normal as soon as possible.

\section{ACKNOWLEDGMENTS}

The Melanoma Research Victoria (MRV) registry, from which data have been used in this study, was originally supported by the Victorian Government through the Victorian Cancer Agency Translational Research Program. The authors thank the following members of MRV for contributing data for this project: David Speakman, David Gyorki, Jonathan Cebon, Damien Kee, John Kelly, Mark Shackleton, Victoria Mar, and Craig Underhill.

\section{DATA AND MATERIAL AVAILABILITY}

The framework has been made publicly available in an online tool: http://stage-shift.personex.nl. All data used to illustrate the framework are included in this publication. The R scripts used to perform the exploratory analyses within the illustration are available from the online tool.

\section{ETHICS APPROVAL AND PATIENT CONSENT}

The Melanoma Research Victoria registry is covered by the following HRECs [PeterMac 07/38; Alfred 284/07; Austin H2008/03230; Border 382/12/1 and Skin Health Institute REC/16/SVHM/149]. Beyond these data, only published summary data were used in the analyses and, hence, no further ethics approval or patient consent was required.

\section{CONFLICT OF INTEREST}

GMc has been a principal investigator for clinical trials with Array Biopharma and Roche/Genentech. All revenues were paid to his institution as reimbursement for trial costs. BJS has served on advisory boards for AstraZeneca, Roche/Genentech, Pfizer, Novartis, Merck, Bristol Myers Squibb, and Amgen. All other authors have declared no conflict of interest.

\section{ORCID}

Koen Degeling (D) https://orcid.org/0000-0001-7487-2491

Nancy N. Baxter (D) https://orcid.org/0000-0003-4793-4620

Jon Emery (iD) https://orcid.org/0000-0002-5274-6336

Mark A. Jenkins (D) https://orcid.org/0000-0002-8964-6160

Fanny Franchini (D) https://orcid.org/0000-0002-4522-3385

Peter Gibbs (D) https://orcid.org/0000-0003-1423-4484

G. Bruce Mann (iD) https://orcid.org/0000-0002-2508-9203

Grant McArthur (iD https://orcid.org/0000-0001-8908-6071

Maarten J. IJzerman (D) https://orcid.org/0000-0001-5788-5805

\section{REFERENCES}

1. Dinmohamed AG, Visser O, Verhoeven RHA, et al. Fewer cancer diagnoses during the COVID-19 epidemic in the Netherlands. Lancet Oncol. 2020;21(6):750-751.

2. Lai A, Pasea L, Banerjee A, et al. Estimating excess mortality in people with cancer and multimorbidity in the COVID-19 emergency. medRxiv. 2020.05.27.20083287.

3. Sud A, Jones ME, Broggio J, et al. Collateral damage: the impact on cancer outcomes of the COVID-19 pandemic. Annal Oncol. 2020;31(8):1065-1074.

4. Olesen F, Hansen RP, Vedsted P. Delay in diagnosis: the experience in Denmark. Br J Cancer. 2009;101(Suppl 2):S5-8.

5. Segelov $\mathrm{E}$, Underhill $\mathrm{C}$, Prenen $\mathrm{H}$, et al. Practical considerations for treating patients with cancer in the COVID-19 pandemic. JCO Oncol Pract;2020:Op2000229.

6. Neal RD, Tharmanathan P, France B, et al. Is increased time to diagnosis and treatment in symptomatic cancer associated with poorer outcomes? Systematic review. Br J Cancer. 2015;112(1):S92.

7. Khorana AA, Tullio $\mathrm{K}$, Elson $\mathrm{P}$, et al. Time to initial cancer treatment in the United States and association with survival over time: an observational study. PloS One. 2019;14(3):e0213209.

8. Coca-Pelaz A, Takes RP, Hutcheson K, et al. Head and neck cancer: a review of the impact of treatment delay on outcome. Adv Ther. 2018;35(2):153-160.

9. Murphy CT, Galloway TJ, Handorf EA, et al. Survival Impact of Increasing Time to Treatment Initiation for Patients With Head and Neck Cancer in the United States. J Clin Oncol. 2016;34(2):169-178.

10. Wanis KN, Patel SVB, Brackstone M. Do moderate surgical treatment delays influence survival in colon cancer?. Dis Colon Rectum. 2017;60(12):1241-1249. 
11. Tørring $\mathrm{ML}$, Falborg $\mathrm{AZ}$, Jensen $\mathrm{H}$, et al. Advanced-stage cancer and time to diagnosis: an International Cancer Benchmarking Partnership (ICBP) cross-sectional study. Eur J Cancer Care (Engl). 2019;28(5):e13100.

12. Connor RJ, Chu KC, Smart CR. Stage-shift cancer screening model. J Clin Epidemiol. 1989;42(11):1083-1095.

13. Chang W, Cheng J, Allaire JJ, Xie Y, McPherson J, shiny: Web Application Framework for R. R package version 1.4.0.2, 2020. https://CRAN. R-project.org/package=shiny

14. R Core Team. R: A Language and Environment for Statistical Computing. Vienna, Austria: R Foundation for Statistical Computing; 2019. https: //www.R-project.org/.

15. Australian Institute of Health and Welfare. Cancer in Australia 2019. Canberra: AlHW; 2019. doi:10.25816/5ebcc7a7fa7e9. Accessed May 18, 2020

16. Goldsbury DE, Yap S, Weber MF, et al. Health services costs for cancer care in Australia: estimates from the 45 and Up Study. PloS One. 2018;13(7):e0201552.

17. Australian Bureau of Statistics. Consumer price index: March Quarter 2020. Canberra, Australia;2020 Report No.: 6401.0. Accessed June 3, 2020.

18. Thursfield V, Farrugia H, Cancer in Victoria: Statistics \& Trends 2017. Cancer Council Victoria, Melbourne 2018. https://www.cancervic.org. $\mathrm{au} / \mathrm{research} /$ registry-statistics/statistics-data

19. Liu W, Dowling JP, Murray WK, et al. Rate of growth in melanomas: characteristics and associations of rapidly growing melanomas. Arch Dermatol. 2006;142(12):1551-1558.

20. Maringe C, Spicer J, Morris M, et al. The impact of the COVID-19 pandemic on cancer deaths due to delays in diagnosis in England, UK: a national, population-based, modelling study. Lancet Oncology. 2020;21(8):1023-1034.

21. Yuan P, Cao JL, Rustam A, et al. Time-to-progression of NSCLC from early to advanced stages: an analysis of data from SEER registry and a single institute. Sci Rep. 2016;6:28477.
22. Bleicher RJ, Ruth K, Sigurdson ER, et al. Time to surgery and breast cancer survival in the United States. JAMA Oncol. 2016;2(3):330339.

23. Lee $\mathrm{Y}-\mathrm{H}$, Kung P-T, Wang $\mathrm{Y}-\mathrm{H}$, Kuo W-Y, Kao S-L, Tsai W-C. Effect of length of time from diagnosis to treatment on colorectal cancer survival: a population-based study. PloS One. 2019;14(1): e0210465.

24. Coumans FA, Siesling S, Terstappen LW. Detection of cancer before distant metastasis. BMC Cancer. 2013;13:283.

25. Tyuryumina EY, Neznanov AA. Consolidated mathematical growth model of the primary tumor and secondary distant metastases of breast cancer (CoMPaS). PloS One. 2018;13(7):e0200148.

26. Samson P, Patel A, Garrett T, et al. Effects of delayed surgical resection on short-term and long-term outcomes in clinical stage I non-small cell lung cancer. Ann Thorac Surg. 2015;99(6):1906-1912.

\section{SUPPORTING INFORMATION}

Additional supporting information may be found online in the Supporting Information section at the end of the article.

How to cite this article: Degeling K, Baxter NN, Emery J, et al. An inverse stage-shift model to estimate the excess mortality and health economic impact of delayed access to cancer services due to the COVID-19 pandemic. Asia-Pac J Clin Oncol. 2021;1-9. https://doi.org/10.1111/ajco.13505 